\title{
Detecting magnetically guided atoms with an optical cavity
}

\author{
Albrecht Haase * Björn Hessmo † and Jörg Schmiedmaye $\ddagger$ \\ Physikalisches Institut, Universität Heidelberg, 69120 Heidelberg, Germany
}

\begin{abstract}
We show that a low finesse cavity can be efficient for detecting neutral atoms. The low finesse can be compensated for by decreasing the mode waist of the cavity. We have used a near concentric resonator with a beam waist of $12 \mu \mathrm{m}$ and a finesse of only 1100 to detect magnetically guided $\mathrm{Rb}$ atoms with a detection sensitivity of 0.1 atom in the mode volume. For future experiments on single atom detection and cavity QED applications, it should be very beneficial to use miniaturized optical resonator integrated on atom chips.

PACS numbers: 03.65.w, 03.75.-b, 42.50.-p, 42.60.Da
\end{abstract}

*Electronic address: haase@physi.uni-heidelberg.de

${ }^{\dagger}$ Electronic address: hessmo@physi.uni-heidelberg.de

${ }^{\ddagger}$ Electronic address: schmiedmayer@physi.uni-heidelberg.de 
It is highly desirable to detect atoms with high efficiency and good spatial resolution both for fundamental physical experiments [1] and for applications in quantum information processing [2]. This task is usually accomplished by using high finesse cavities, where the photons interact strongly with the atoms. In such experiments it has been possible to monitor the motion of single atoms inside a high finesse $\left(\mathcal{F}>2 \times 10^{5}\right)$ cavity[3]. By performing feedback to the optical beam passing through the resonator it has been possible to control the atomic motion inside the cavity to form bound atom-photon states. This also requires a high finesse $\left(\mathcal{F}>4 \times 10^{5}\right)$ resonator $[4]$.

In this article we wish to explore the possibilities to perform atom detection using optical cavities with moderate finesse [5]. The main result is that the finesse $\mathcal{F}$ is not the most important aspect of cavity assisted detection schemes, equally important is the ratio between the atomic absorption cross section $\sigma_{\text {atom }}=3 \lambda^{2} / 2 \pi$ and the beam cross section inside the cavity $A=\frac{\pi}{4} w_{0}^{2}$.

For each round trip the photon is absorbed with a probability $\sigma_{\text {atom }} / A$. A figure of merit for absorption inside the cavity is therefore

$$
C_{1}=\frac{\mathcal{F}}{2 \pi} \frac{\sigma_{\text {atom }}}{A} .
$$

where $\frac{\mathcal{F}}{2 \pi}$ is the number of round trips for a photon. This quantity is identical to the cooperativity parameter $C_{1}=\frac{g_{0}^{2}}{2 \kappa \gamma}$, which relates the time scales of the coherent dynamics of the coupled system $g_{0}^{-1}$ to the time scales of incoherent decays of cavity field $\kappa^{-1}$ and atomic excitation $\gamma^{-1}$. This is also related to the Purcell factor $\eta=2 C_{1}$ that determines the enhancement of the spontaneous emission rate into the cavity mode over the free space value[1, 6]. Looking at Eqn. (11) one clearly sees that a reduced cavity mode waist can compensate for a small cavity finesse.

In this spirit it has been proposed by Horak et al. that a single atom detection in low finesse can be achieved by strongly focussing the cavity mode [5]. When the cooperativity parameter is smaller than one and the atomic saturation is low the signal-to-noise ratio for a single atom detection becomes

$$
S=\sqrt{j_{\text {in }} \tau} \frac{\kappa_{T}}{\kappa} C_{1}
$$

where $j_{\text {in }}$ is the incident photon flux, $\tau$ the measurement interval, $\kappa_{T}$ the mirror transmission rate, and $\kappa$ the overall cavity decay rate [5]. For a fixed measurement time an increased signalto-noise ratio can be obtained by increasing the cooperativity parameter. This can be done 
by increasing the cavity finesse, or by decreasing the beam waist. Here we explore the latter case, when the beam cross section is reduced.

To achieve this we use a nearly concentric cavity geometry. Our cavity was formed by two identical mirrors with radius of curvature $R$ separated by a distance $L$. The beam waist $w_{0}$ in the cavity center is given by $w_{0}^{2}=\frac{\lambda}{2 \pi} \sqrt{L(2 R-L)}$. The concentric geometry occurs when the mirror separation $L$ approaches the value $2 R$. The waist size $w_{0}$ becomes small but the beam size on the cavity mirrors $w^{2}=\frac{R \lambda}{\pi} \sqrt{\frac{L}{2 R-L}}$ becomes large as one approaches the concentric limit.

A large mirror spot size requires very uniform mirrors as deviations from a spherical mirror shape will lower the optical finesse drastically. Furthermore, as the concentric point is approached, the cavity also becomes extremely sensitive to misalignments and vibrations. For more details on this cavity we refer to Siegman [7].

Table 【 summarizes parameters for nearly concentric cavities and shows that it is not stringently necessary to have $C_{1}>1$ to detect the presence of a single atom within the cavity mode.

These considerations all concern the coupling of a single atom to a cavity. This can be generalized to the many atom case by introducing a many-atom cooperativity parameter $C=N_{\text {eff }} C_{1}$, where $N_{\text {eff }}$ is an effective number of atoms in the cavity mode [8], which takes into account the spatial dependency of the coupling constant $g(\vec{r})=g_{0} \psi(\vec{r})$, given by the cavity mode function $\psi(\vec{r})$, and the atomic density distribution $\rho(\vec{r})$. The fraction of the total atom number $N$ which is maximally coupled to the cavity mode is given by the overlap integral of both functions

$$
N_{\text {eff }}=N \int d^{3} r \rho(\vec{r})|\psi(\vec{r})|^{2} .
$$

The absorptive and dispersive effect of the atoms on the cavity amplitude [5] scale linearly with this effective atom number as long as the atomic saturation is low.

To explore atom detection with low finesse cavities experimentally, we built a magnetooptical trap (MOT) for ${ }^{85} \mathrm{Rb}$ atoms approximately $20 \mathrm{~mm}$ above the cavity center (see Fig. (1). It contained $\sim 10^{7}$ atoms at a temperature of $35 \mu \mathrm{K}$. From the MOT we proceeded in two different ways. Either we switched off the trap completely and monitored the atomic cloud as it fell freely through the cavity, or we transfered the atoms to a magnetic wire guide that channeled the atoms through the cavity [9]. The magnetic guide was formed by a current-carrying wire, attached to the cavity mounting in vertical direction (see Fig. 1b) 
and a homogeneous magnetic bias field in the direction of the optical axis of the cavity. In this configuration a two dimensional quadrupole guide was formed. The depth, confinement, and position was controlled by varying the wire current and the magnetic bias field [9, 10].

To keep the cavity aligned, we mounted one of the mirrors on a piezoelectric tripod that allowed us to adjust the optical axis of the cavity. This mirror was aligned to keep the $\mathrm{TEM}_{00}$ mode centered on the cavity axis. The second mirror was mounted on a translating piezoelectric stage for wavelength tuning. Feedback to this piezo actuator was generated using the Pound-Drever-Hall technique [11] to lock the cavity on the laser beam passing through the cavity. Figure 2 illustrates how the cavity finesse was reduced as the concentric point was approached for our cavity. We used two mirrors with $R=10 \mathrm{~mm}$ and transmission $T=10^{-3}$. For a mirror separation far less than the concentric limit these mirrors yielded a finesse of 3000 . This finesse dropped to 1100 when the separation was $70 \mu \mathrm{m}$ from the concentric point as discussed in the introduction. The cavity mode waist was $12.1 \mu \mathrm{m}$ for this separation.

We monitored the light intensity transmitted through with an amplified photodiode for high light intensities or with a photomultiplier tube (PMT) for low light intensities. The PMT provided a near shot-noise limited detection. The low-noise electronic amplification limited the detection bandwidth to $20 \mathrm{kHz}$. The main source of technical noise in our setup was due to mechanical vibrations of the vacuum chamber that held the cavity.

The drop in the cavity transmission signal from freely falling atoms is plotted in Fig. [3a). Different curves manifest different pump powers corresponding to empty cavity transmissions between $1 \mathrm{pW}$ and $60 \mathrm{pW}$. The atom number in the MOT is $1.5 \times 10^{7}$, the signal drops by $90 \%$ as long as the atomic transition is not saturated (Fig. 3b). Fitting this data with the theoretical model[8], one obtains an effective atom number $N_{\text {eff }}=2.5 \pm 0.5$. This was consistent with an independent atom number measurement based on florescence imaging. To explore the sensitivity limit of the cavity detector, the atom number in the MOT was successively reduced until the signal drop due to the atoms was overshadowed by the noise.

When the MOT contained $3.5 \times 10^{5}$ atoms this produced a signal drop of approximately $10 \%$. We consider this to be the resolution limit. A theoretical fit results in an effective atom number of $N_{\text {eff }}=0.1 \pm 0.05$.

As a next step, atoms were magnetically guided to the cavity center using the wire guide (see Fig. (1). By changing the current in the guiding wire the overlap between the atoms and 
the cavity mode could be adjusted. In Fig. 4 we plot the cavity transmission as the position of the magnetic guide is varied across the cavity mode. As the atomic overlap with the cavity mode was increased, we observed a increased drop in cavity transmission. From the duration of the transmission drop the temperature of the guided atoms could be determined to be $25 \mu \mathrm{K}$.

The density distribution for the atoms was much larger than the Rayleigh volume of the cavity, consequently it was not possible to distinguish individual atoms in the guide using our low finesse cavity. This cavity would however show a detectable change in the transmission signal if a single atom would cross the region of maximum coupling as $N_{\text {eff }}$ can be as small as 0.1. The precision in the positioning can be improved using magnetic microtraps, e.g. produced by atom chip surface traps[12]. On the atom chip one can also build small integrated cavities [13] with mode waists as small as $2 \mu \mathrm{m}$. This relaxes the requirements on the finesse even further [5]. To achieve the same detection sensitivity with a beam waist of $2 \mu \mathrm{m}$ a finesse of 40 is enough.

To conclude, we have illustrated that it is possible to detect magnetically guided atoms using a low finesse cavity with small mode waist. The small waist allowed us to detect atoms with high sensitivity, as illustrated in Fig. 3. We also show that high spatial resolution can be achieved. We demonstrate this by detecting magnetically guided atoms, as illustrated in Fig. 4. A natural development would be to miniaturize the cavity even further and integrate it on an atom chip [5, 13, 14, 15, 16].

We gratefully acknowledge valuable discussions with P. Horak, T. Fernholz, and M. Wilzbach. Funding was provided by Landesstiftung Baden-Württemberg, Forschungsprogramm Quanteninformationsverarbeitung and the EU-program MRTN-CT-2003-50532.

[1] P. Berman, Cavity Quantum Electrodynamics (Academic press, San Diego, 1994).

[2] D. P. D. Vincenzo, Forts. der Physik 48, 771 (2000).

[3] H. Mabuchi, J. Ye, and H. Kimble, Appl. Phys. B 68, 1095 (1999).

[4] P. Pinkse, T. Fischer, P. Maunz, and G. Rempe, Nature 404, 365 (2000).

[5] P. Horak, B. G. Klappauf, A. Haase, R. Folman, J. Schmiedmayer, P. Domokos, and E. A. Hinds, Phys. Rev. A 67, 043806 (2003). 
[6] E. M. Purcell, Phys. Rev. 69, 681 (1946).

[7] A. E. Siegman, Lasers (University Science Books, Mill Valley, 1986).

[8] A. Haase, Ph.D. thesis, Universität Heidelberg (2005).

[9] J. Denschlag, D. Cassettari, and J. Schmiedmayer, Phys. Rev. Lett. 82, 2014 (1999).

[10] A. Haase, D. Cassettari, B. Hessmo, and J. Schmiedmayer, Phys. Rev. A 64, 043305 (2001).

[11] R. Drever, J. Hall, F. Kowalski, J. Hough, G. Ford, A. Munley, and H. Ward, Appl. Phys. B 31, 97 (1983).

[12] R. Folman, P. Krüger, J. Schmiedmayer, J. Denschlag, and C. Henkel, Adv. At. Mol. Phys. 48, $263(2002)$.

[13] X. Liu, K. H. Brenner, M. Wilzbach, M. Schwarz, T. Fernholz, and J. Schmiedmayer (2005), Appl. Optics, in print.

[14] D. Armani, T. Kippenberg, S. Spillane, and K. Vahala, Nature 421, 925 (2003).

[15] B. Lev, K. Srinivasan, P. Barclay, O. Painter, and H. Mabuchi, Nanotechnology 15 (2004).

[16] Z. Moktadir, E. Koukharenka, M. Kraft, D. M. Bagnall, H. Powell, M. Jones, and E. A. Hinds, J. Micromech. Microeng. 14, 82 (2004). 


\begin{tabular}{|c|c|c|c|c|c|c|}
\hline$L$ & $w_{0}$ & $\mathcal{F}$ & $g_{0}$ & $\kappa$ & $C_{1}$ & $S_{\max }$ \\
\hline$[\mathrm{mm}]$ & {$[\mu \mathrm{m}]$} & & $2 \pi \times[\mathrm{MHz}]$ & $2 \pi \times[\mathrm{MHz}]$ & & \\
\hline 19.0 & 23.3 & 1000 & 1.6 & 3.9 & 0.1 & 2.8 \\
19.93 & 12.1 & 1000 & 3.0 & 3.8 & 0.4 & 6.1 \\
19.99 & 7.5 & 1000 & 4.9 & 3.8 & 1.1 & 13.2 \\
19.99 & 7.5 & 300 & 4.9 & 12.5 & 0.3 & 5.3 \\
\hline
\end{tabular}

TABLE I:

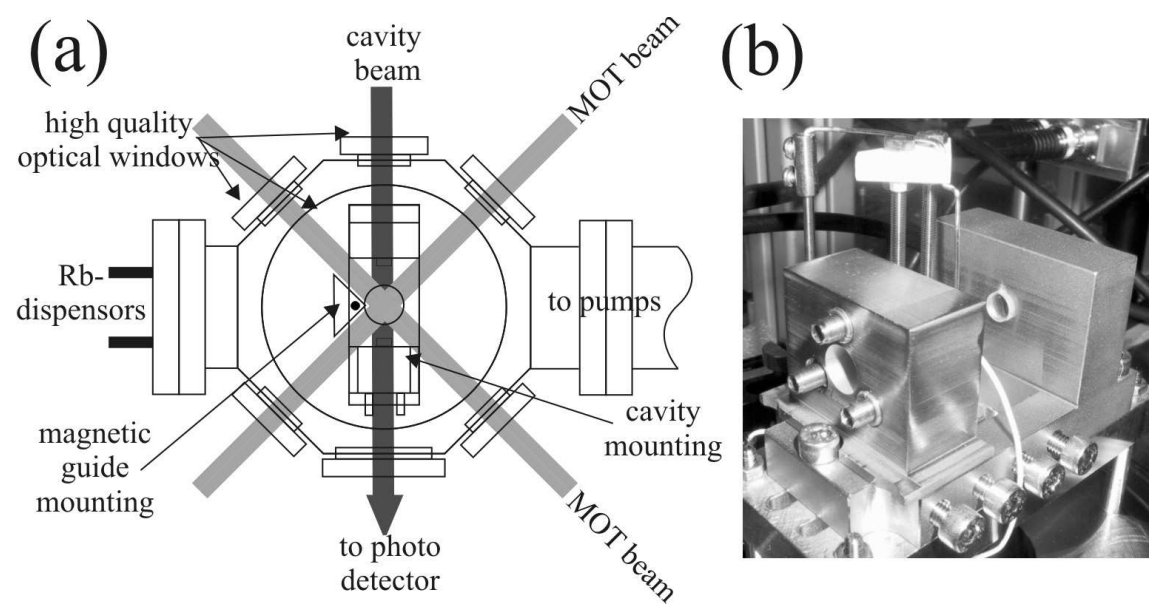

FIG. 1:

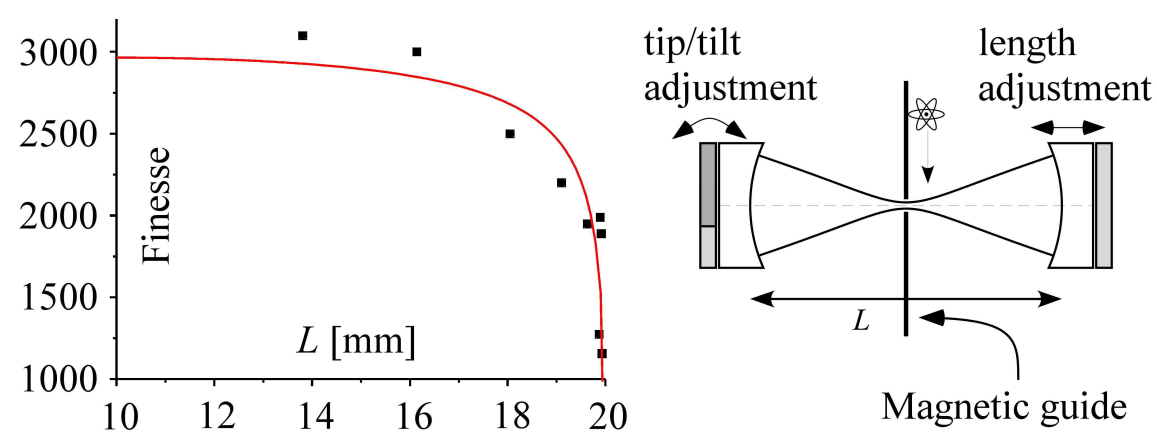

FIG. 2: 


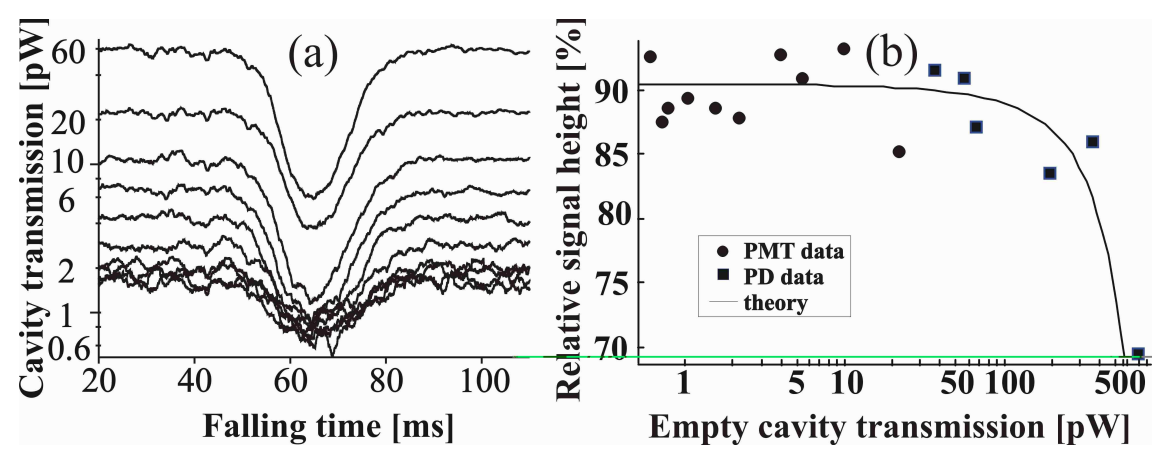

FIG. 3:

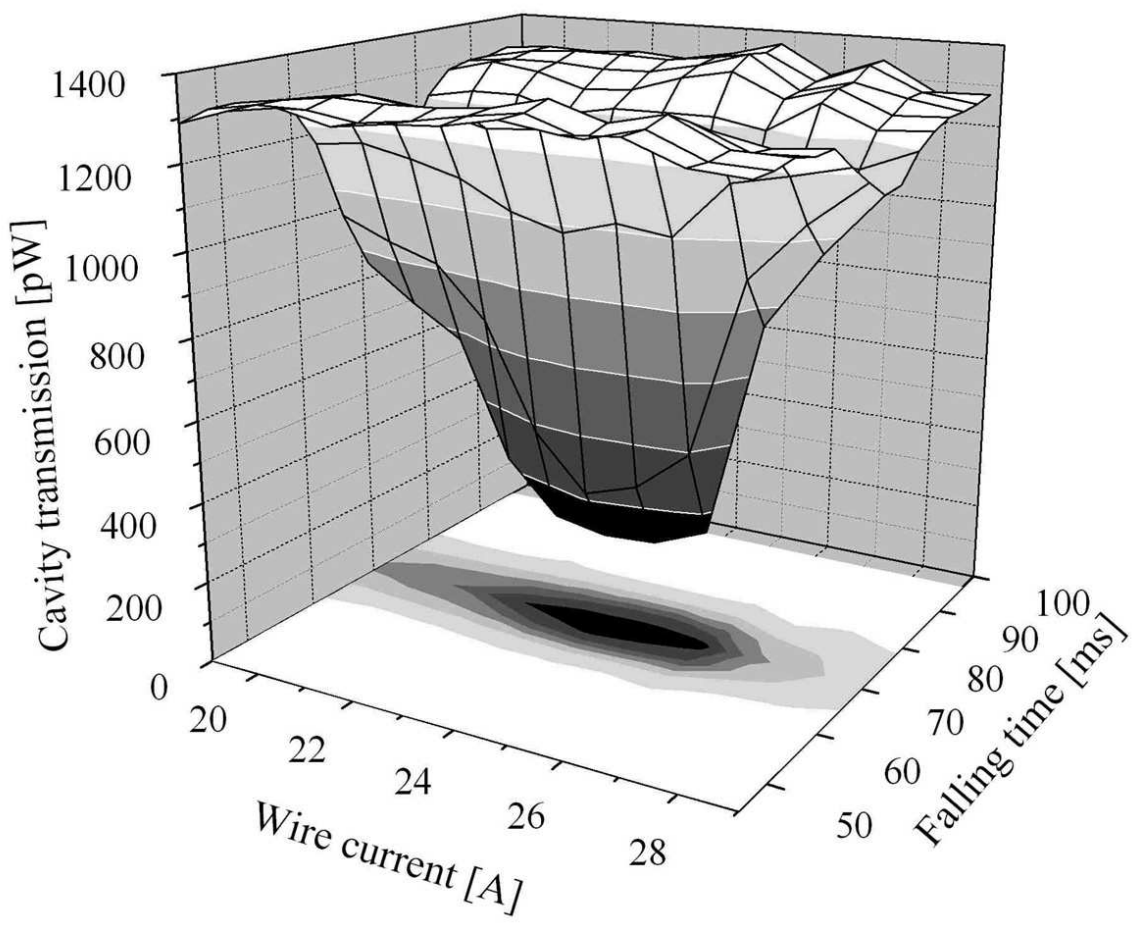

FIG. 4: 
- Caption Tab. I: In the table the cooperativity parameters and maximum signalto-noise ratios for various cavity geometries close to the concentric cavity limit at $L=2 R=20 \mathrm{~mm}$ are listed. The atomic decay rate is that of the rubidium D2 transition and $\tau=10 \mu \mathrm{s}$.

- Caption Fig. 1: (a) Schematic drawing of the experimental chamber which contains a magneto-optical trap, the quasi-concentric cavity and a magnetic wire guide. (b) Picture of the cavity mounting including guiding wire.

- Caption Fig. 2: a) The finesse of the cavity decreases as the concentric point is approached. The curve is calculated from the cavity geometry and mirror specifications. b) Schematic description of the cavity. The two mirrors with $10 \mathrm{~mm}$ radius of curvature are mounted on piezo electric actuators for alignment. One of the mirrors is can be tilted to keep the optical axis of the cavity fixed. The other mirror can be translated for frequency tuning. Atoms can be magnetically guided through the cavity.

- Caption Fig. 3: (a) Cavity transmission signal for atoms dropped from a MOT. Different curves correspond to cavity pump powers from 1-60pW. The signal has been averaged over $2.5 \mathrm{~ms}$ for better visualization. (b) Relative drop of the signal due to the atoms in (a). The circles (squares) come from measurements with a PMT (photodiode) for different light intensities. The black line is calculated numerically.

- Caption Fig. 4: Cavity transmission signal from atoms being magnetically guided through the cavity mode. The position of the potential minimum is linearly dependent on the wire current. 